\title{
An annual review of the remarkable advances in lung cancer clinical research in 2019
}

\author{
Bo Cheng ${ }^{1 \#}$, Shan Xiong ${ }^{1 \#}$, Caichen Li $^{1 \#}$, Hengrui Liang ${ }^{1}$, Yi Zhao ${ }^{1}$, Jianfu Li $^{1}$, Jiang Shi ${ }^{1}$,imin Ou ${ }^{1}$, \\ Zisheng Chen ${ }^{1,2}$, Peng Liang ${ }^{1}$, Wenhua Liang ${ }^{1}$, Jianxing $\mathrm{He}^{1}$ \\ ${ }^{1}$ Department of Thoracic Surgery and Oncology, the First Affiliated Hospital of Guangzhou Medical University, State Key Laboratory of Respiratory \\ Disease \& National Clinical Research Center for Respiratory Disease, Guangzhou 510120, China; ${ }^{2}$ Department of Respiratory Medicine, the Sixth \\ Affiliated Hospital of Guangzhou Medical University, Qingyuan People's Hospital, Qingyuan 511500, China \\ Contributions: (I) Conception and design: B Cheng, J He, W Liang; (II) Administrative support: J He, W Liang; (III) Provision of study materials or \\ patients: S Xiong, C Li; (IV) Collection and assembly of data: All authors; (V) Data analysis and interpretation: All authors; (VI) Manuscript writing: \\ All authors; (VII) Final approval of manuscript: All authors. \\ \#These authors contributed equally to this work. \\ Correspondence to: Jianxing He, MD; Wenhua Liang, MD. Department of Thoracic Surgery and Oncology, the First Affiliated Hospital of Guangzhou \\ Medical University, State Key Laboratory of Respiratory Disease, National Clinical Research Center for Respiratory Disease, 151, Yanjiang Road, \\ Guangzhou 510120, China. Email: drjianxing.he@gmail.com; liangwh1987@163.com.
}

\begin{abstract}
In recent years, lung cancer has become the most common cancer and the leading cause of deaths attributed to cancer. In China, lung cancer is the third leading cause of death after strokes and ischemic heart disease. This review aimed to provide an up-to-date summary of studies in regard to lung cancer in 2019 and to present the remarkable progress seen in lung cancer clinical research. A systematic search of PubMed and Web of Science for research published in 2019 was conducted using the search terms "lung cancer", "early stage", "advanced", "diagnosis", "treatment”, or any combination of these terms. We selected 56 studies that we considered to be significant and have presented their major findings. In summary, survival among lung cancer patients was significantly improved compared with historical controls, turning lung cancer from an incurable disease into a chronic disease. In addition, treating lung cancer has become increasingly comprehensive, diversified, and individualized. Exploring the accurate biomarkers in immunotherapy and the mechanism of drug resistance in targeted therapy constitutes the greatest challenge at this stage.
\end{abstract}

Keywords: Lung cancer; clinical trial; chemotherapy; immunotherapy; targeted therapy

Submitted Jan 21, 2020. Accepted for publication Feb 27, 2020.

doi: $10.21037 /$ jtd.2020.03.11

View this article at: http://dx.doi.org/10.21037/jtd.2020.03.11

\section{Introduction}

The diagnosis and treatment of lung cancer became a hot spot of research due to the disease's increased morbidity and mortality in recent years. Therefore, more and more novel diagnosis methods for early-stage lung cancer and plenty of clinical trials have been conducted. Over past 12 years, China has seen a significant increase in the 5 -year survival rates of various cancers, among which the 5-year survival rate of lung cancer has increased by an average of $1.3 \%$ every year and has achieved $19.7 \%$ so far (1). In terms of different patient characteristics, a series of treatment modes have been adopted, such as combination therapy, sensitization of drugs, and targeted therapy on various driver mutations. The aim of this review is to provide an up-to-date summary of lung cancer studies in 2019 and to present the remarkable progress made in lung cancer clinical research.

\section{Methods}

A systemic search of PubMed and Web of Science was 
conducted to find studies or conference abstracts published in English in the period between January 1, 2019 and December 31, 2019. The search terms included "lung cancer", "early stage", "advanced", "diagnosis", "treatment", or any combination of these terms. The articles published in The New England Fournal of Medicine (NEFM), The Lancet, $7 A M A, B M \mathcal{F}$, and some other journals in the oncology field, as well as the abstracts reported in the conferences of the American Society of Clinical Oncology (ASCO), the World Conference on Lung Cancer (WCLC), and the European Society for Medical Oncology (ESMO) were included. Additionally, we reviewed in detail the survival of patients, the efficacy of drugs and drug-related adverse events in each enrolled study.

\section{Results}

\section{Early-stage lung cancer}

The trends and risk of morbidity in lung cancer Lung cancer has become the third leading cause of death in China

In June 2019, The Lancet published China's burden of disease data online, covering a period of nearly 30 years (2). The data, which was reported by Professor Xiaofeng Liang from the Chinese Center for Disease Control and Prevention, revealed lung cancer to have become the third leading cause of death in China after strokes and ischemic heart disease (IHD). Between 1990 to 2017, increases of $28.2 \%$ and $12.6 \%$, respectively, were seen in agestandardized mortality and years of life lost (YLLs) in lung cancer, leading to a change in ranking from 13th in 1990 to third in 2017.

This trend and current situation suggest that the prevention and control of lung cancer needs to be carried out with reference to the management mode of common diseases such as diabetes and hypertension, including the popular science and screening activities for lung cancer which are increasingly valued in China. How to balance the goals of early diagnosis and concerns regarding the health economy will be an important challenge.

Dietary fiber and yogurt consumption were associated with reduced risk of lung cancer

In October 2019, FAMA Oncology published a meta-analysis of data from ten prospective cohort studies performed by Xiao-Ou Shu's team at Vanderbilt University (3), which involved a total of 1.44 million people and 8.6 years of follow-up. The results showed that dietary fiber and yogurt consumption were associated with a lower risk of lung cancer. The two groups with the highest intake of fiber and yogurt had a lower risk (17\% and 19\% lower, respectively) of lung cancer than the group with the lowest intake. For individuals who reported high yogurt consumption along with the highest fiber intake, the risk of lung cancer was reduced by $33 \%$ compared with those who did not consume yogurt and had the lowest fiber intake. In addition, young people may benefit more from the consumption of dietary fiber and yogurt.

\section{Early screening and diagnosis of lung cancer} Genetic risk of lung cancer in Chinese and its measurement In July 2019, a large multicenter study of the Chinese population conducted by Professor Hongbing Shen and his team from Nanjing Medical University was published in The Lancet Respiratory Medicine (4). This study systematically identified 19 susceptibility loci to be significantly associated with risk of non-small cell lung cancer (NSCLC) in the Chinese population through a cohort involving nearly 55,000 people and built a polygenic risk score (PRS) for Chinese people based on it. Meanwhile, this research, which was verified by an independent prospective cohort of about 100,000 individuals from the China Kadoorie Biobank $(\mathrm{CKB})$, indicated that the incidence of lung cancer in the highest risk group was nearly double that of the lowest risk group. Moreover, PRS was an independent, effective predictive indicator of risk beyond age and smoking, which could provide an important reference for the screening and accurate prevention of lung cancer for high-risk individuals.

\section{Liquid biopsy (LBx) is expected to be used as an early diagnosis tool for lung cancer}

The preliminary results of Circulating Cell-free Genome Atlas (CCGA) Study were released at the conference of the ASCO and the ESMO $(5,6)$. By combining three prototype assays [whole-genome bisulfite (WGBS), wholegenome sequencing (WGS), and targeted (507 gene) sequencing], signals from 12 cancers including lung cancer can be detected at an early stage. The overall detection rate (sensitivity) of this method for all stages and types of cancers was $55 \%$, and the tissue of origin (TOO) localization could also be identified with high accuracy. The detection results of early-stage cancer (stage I-III) were favorable, with a sensitivity of $32 \%, 76 \%$, and $85 \%$ for stages I, II, and III, respectively. This is consistent with the preliminary results relating to lung cancer of the CCGA study announced by ASCO annual meeting last year, which showed a diagnostic sensitivity of $38-51 \%$ for stage I lung cancer. 
In addition, Professor Jianxing He and his team from Guangzhou Institute of Respiratory Health developed a non-invasive diagnostic tool for early-stage lung cancer using high-throughput targeted DNA methylation sequencing of circulating tumor DNA (ctDNA), which had a sensitivity of more than $70 \%$ for stage I lung cancer (7). Moreover, this assay was being validated through two national multicenter studies (NCT03181490 and NCT03651986) in current stage, including the "Thunder Project" which involves more than 10,000 cases.

\section{The treatment of stage I-III lung cancer}

\section{Immunotherapy joins the "neoadjuvant family"}

Three important neoadjuvant immunotherapy studies were presented at the ASCO annual meeting in June 2019: LCMC3 (8), NEOSTAR (9), and NADIM (10).

(I) The LCMC3 study assessed the efficacy and safety of atezolizumab used as neoadjuvant therapy for patients with stages IB to selected IIIB resectable NSCLC. The interim analysis showed that $7 \%$ of patients achieved partial response (PR), 18\% achieved major pathological response (MPR), and $5 \%$ achieved pathological complete response (pCR). Pathological response was also observed in some patients with positive epidermal growth factor receptor (EGFR)/ALK mutations. Neither PD-L1 expression nor tumor mutational burden (TMB) level was correlated with pathological response. This regimen was tolerated.

(II) NEOSTAR confirmed that nivolumab (NV) plus ipilimumab showed better efficacy as a neoadjuvant therapy for resectable NSCLC patients than NV monotherapy. Patients received neoadjuvant therapy for three cycles before surgery and underwent surgery within three to six weeks after treatment. The results revealed that the MPR rate was $17 \%$ in the monotherapy group vs. $33 \%$ in the combination group, and the pCR rate was $9 \%$ vs. $21 \%$ in the monotherapy and combination groups, respectively. For imaging evaluation, the objective response rate (ORR) was $22 \%$ and $19 \%$ in the monotherapy group and the combination group, respectively, and the ORR was positively correlated with MPR $(\mathrm{P}<0.001)$. Subgroup analysis presented that the higher expression of PD-L1, the benefit of imaging, and the pathological response of patients would be greater. Additionally, this combination could increase the lymphocyte infiltration of the tumor and enhance cell diversity and reactivityrelated function. No unacceptable toxicity or increased perioperative morbidity or mortality was found.

(III) NADIM was the first multicenter study on conventional chemotherapy (CT) combined with immuno-oncology agent (IO) in a neoadjuvant setting for stage IIIA patients. Participants received three cycles of $\mathrm{NV}+$ paclitaxel + carboplatin as preoperative treatment, followed by adjuvant $\mathrm{NV}$ treatment for 1 year after the surgery. The chemotherapy combined with immunology was well tolerated, and 41 patients ultimately underwent surgery, with $\mathrm{R} 0$ resection in all cases. Downstaging was seen in $90 \%$ of cases after neoadjuvant $\mathrm{CT}+\mathrm{IO}$, the MPR rate was $83 \%$, and the pCR rate was $71 \%$. According to RECIST (Response Evaluation Criteria in Solid Tumors), the imaging $\mathrm{PR}$ rate was $71 \%$, and the $\mathrm{CR}$ rate was $7 \%$. No patients withdrew from the study before surgery due to progressive disease (PD) or safety problems.

Neoadjuvant immunotherapy, especially combined with chemotherapy, achieved a surprising pathological response rate, which will largely change the treatment model of early- and middle-stage lung cancer. However, a series of questions relating to, for example, response evaluation criteria, surgery time, and postoperative treatment choices also surfaced and need to be answered.

\section{Treatment strategy options for early-stage lung cancer}

(I) Stereotactic body radiotherapy (SBRT) was widely used to treat inoperable stage-I NSCLC. The Lancet Oncology published the phase 3 randomised controlled trial (RCT) "TROG 09.02 CHISEL", the results of which indicated that SBRT compared to standard radiotherapy could reduce the local recurrence rate $(14 \%$ vs. $31 \%$, respectively), which supports the use of SBRT in treating early-stage NSCLC (11).

(II) In relation to adjuvant treatment options for stage-I NSCLC patients, as defined by the eighth edition TNM staging system, Jianxing $\mathrm{He}$ and Wenhua Liang's research group from Guangzhou Institute of Respiratory Health established a risk prediction model based on data from a large sample size, which was published on Annals of Surgical Oncology (12). This research found that $21.7 \%$ of stage IB patients $(7.5 \%$ of all stage I) 
were categorized into the high-risk of recurrence group, and verified that these patients can benefit from chemotherapy. Furthermore, in regard to the optimal treatment for stage IIIA-N2 NSCLC, the same team published a network meta-analysis based on 18 RCTs in The Annals of Thoracic Surgery (13), finding that neoadjuvant chemotherapy followed by surgery and adjuvant chemotherapy or radiotherapy holds the greatest potential to become the optimal treatment with the best efficacy and safety. This paper was included in the recommended literature on the F1000Prime platform.

The treatment of locally advanced NSCLC Immunology consolidation therapy after concurrent chemoradiotherapy

The PACIFIC study is a milestone to rewrite the therapeutic method of locally advanced NSCLC, the results of which were published in NEFM for twice in 2018 and confirmed that treatment with PD-L1 antibody durvalumab after concurrent chemoradiotherapy for unresectable stage III NSCLC can significantly prolong progression free survival (PFS) and overall survival (OS) compared with the placebo group. The PACIFIC study presented the 3-year updated OS data at the 2019 ASCO annual meeting (14). The median duration of follow-up was 33.3 months, and the median OS (mOS) of the treatment group was significantly longer than that of the placebo group [not reached (NR) $v s$. 29.1 months]. The 3-year OS rate in these two groups was $57 \%$ vs. $43.5 \%$, respectively. Compared with the placebo group, patients in the treatment group received subsequent therapies for a longer period of time, with the first subsequent treatment continuing for twice as long (21.2 vs. 10.4 months), and the second subsequent treatment lasting for 30.2 vs. 17.8 months.

Furthermore, the PACIFIC study updated the information of patient-reported outcomes (PROs) in The Lancet Oncology (15), proving that with the consolidation therapy of durvalumab, OS could be greatly improved in patients with unresectable stage III NSCLC and patients' quality of life was not compromised.

\section{Immunotherapy plus concurrent chemoradiotherapy}

The current concerned point is whether immunotherapy can be used concurrently with chemoradiotherapy. The treatment of atezolizumab combined with concurrent chemoradiotherapy had favorable safety for locally advanced NSCLC patients, as reported at ASCO annual meeting 2019, suggesting that concurrent chemoradiotherapy plus immunotherapy does not increase toxicity compared with chemoradiotherapy followed by immunotherapy. At present, the PACIFIC2 study is also under way, and the impact on efficacy due to bringing immunotherapy forward to the same stage as chemoradiotherapy is anticipated.

Whether patients with locally advanced NSCLC need prophylactic cranial irradiation (PCI)

An update of a prospective, randomized, multicenter phase 3 study of locally advanced NSCLC patients (RTOG 0214 trial) was published in $7 A M A$ Oncology (16). In patients without disease progression after local therapy, despite PCI therapy significantly decreasing the incidence of brain metastasis (BM) and remarkably improving disease-free survival (DFS) compared with the observation group, there was no significant difference in terms of OS (the 5- and 10 -year rates were $24.7 \%$ and $17.6 \%$ vs. $26.0 \%$ and $13.3 \%$, respectively).

\section{Advanced lung cancer}

First-line immunotherapy for advanced lung cancer IO monotherapy

\section{(I) KEYNOTE-024}

Keynote-024 updated 3-year OS data at the WCLC conference 2019 and showed that the superior efficacy of PD-1 antibody monotherapy compared to CT. There was a significant difference in the 3 -year OS rate between the treatment group and the control group (43.7\% vs. $24.9 \%$, respectively) (17).

\section{(II) KEYNOTE-042}

This important phase 3 study was formally published in The Lancet, and its results confirmed that for locally advanced or metastatic NSCLC patients without sensitizing EGFR/ALK alterations and with PD-L1 positive [tumor proportion score (TPS) $\geq 1 \%$ ], first-line pembrolizumab treatment could obviously extend the OS compared to chemotherapy (16.7 vs. 12.1 months; HR, 0.81; 95\% CI, $0.71-0.93 ; \mathrm{P}=0.0018)$ and was safer (18).

\section{(III) IMpower 110}

The interim OS analysis announced on the ESMO congress demonstrated that, compared to standard chemotherapy, atezolizumab significantly improved the mOS (20.2 vs. 13.1 months, respectively; HR, 0.59; 95\% CI, 0.40-0.89; $\mathrm{P}=0.0106)$ of untreated stage IV patients with $\mathrm{PD}-\mathrm{L} 1$ high expression (TC3/IC3-WT). Median PFS was 8.1 vs. 5.0 months (HR, 0.63; 95\% CI, 0.45-0.88; $\mathrm{P}=0.007$ ) for atezolizumab and standard chemotherapy, respectively. ORR and duration of response (DoR) had notable benefits 
with atezolizumab (19).

Throughout the current research data for IO monotherapy, the level of patients' PD-L1 expression has been the key to selecting IO monotherapy. Existing evidence demonstrates that IO monotherapy should be preferentially considered for patients with PD-L1 high expression $(\geq 50 \%)$, whereas a combination of immunoand chemo-therapy should be considered for patients with PD-L1 expression $<50 \%$.

\section{The long-term survival outcome of PD-1 monoclonal antibody treatment}

(I) The Lancet Oncology published the long-term survival outcome of $\mathrm{NV}$ as a second-line treatment for advanced NSCLC patients (20). A pooled analysis from the Checkmate 017 and 057 studies showed the 4-year OS rate with NV was $14 \%$ for all patients, and $19 \%$ for those with positive PD-L1 expression (at least 1\%), which was far higher than the $5 \%$ of docetaxel chemotherapy. This confirmed that PD-1 antibody immunotherapy can achieve a clinical cure for some patients.

(II) The ASCO annual meeting presented the longterm outcomes of the KEYNOTE-001 trial (21). With pembrolizumab monotherapy treatment, the estimated 5-year OS rates were 23.2\% for treatment-naive advanced NSCLC patients and $15.5 \%$ for patients who had been previously treated.

\section{IO plus chemotherapy}

(I) KEYNOTE-189 (22)

At the 2019 ASCO annual meeting, the KEYNOTE-189 study updated the OS results after follow-up time was extended and, for the first time reported the results of PFS2 (progression after the next line of therapy). For nonsquamous NSCLC, persistent OS and PFS benefit was still observed in the combination arm of pembrolizumab and chemotherapy. For the combination and control arms, mOS was 22.0 vs. 10.7 months, respectively (HR, 0.56; 95\% CI, 0.45-0.70; $\mathrm{P}<0.00001$ ), and median PFS (mPFS) was 9.0 vs. 4.9 months, respectively (HR, 0.48; 95\% CI, 0.40-0.58; $\mathrm{P}<0.00001)$. In the intention-to-treat (ITT) population, PFS2 in the combination arm was significantly higher than in chemotherapy alone arm. In these two groups, the mPFS2 was 17.0 vs. 9.0 months, respectively (HR, 0.49; 95\% CI, $0.40-0.59 ; \mathrm{P}<0.00001)$. The benefit of all endpoints was independent of the PD-L1 TPS level of patients.

\section{(II) KEYNOTE-407 (23)}

The study's updated outcome at the ESMO congress 2019 indicated that for squamous NSCLC, first-line treatment with pembrolizumab plus chemotherapy significantly prolonged PFS2. In the chemotherapy alone group, 49.1\% of the patients received the subsequent treatment of PD-1/ PD-L1 antibody, and the combination of pembrolizumab and chemotherapy still brought continuous OS benefit to patients. In the ITT population, mOS attained 17.1 months (range, 14.4 to 19.9 months), compared to 11.6 months (range, 10.1 to 13.7 months) in the chemotherapy alone group, and the mortality risk was reduced by $29 \%$ with combination treatment (HR, 0.71; $5 \%$ CI, 0.58-0.88). In comparison with chemotherapy alone, combination treatment also significantly improved the PFS of patients. Regardless of the level of PD-L1 expression of patients, pembrolizumab plus chemotherapy continued to demonstrate improved end points including OS, PFS, ORR, and PFS2 as the first-line treatment, compared with chemotherapy alone for advanced squamous NSCLC, with a manageable safety profile.

\section{(III) IMpower130}

The results published in The Lancet Oncology showed a significant and clinically meaningful improvement in OS and PFS with atezolizumab plus chemotherapy versus chemotherapy alone as first-line treatment for patients with stage IV nonsquamous NSCLC and no ALK or EGFR mutations (24), with acceptable safety and tolerability. This was the first PD-L1 inhibitor to be successfully used as firstline treatment for advanced NSCLC.

These study results further consolidated the status of pembrolizumab as well as atezolizumab combined with chemotherapy as the first-line standard treatments for advanced NSCLC patients with negative driver genes, and suggested once again that the earlier the use of immunotherapy, the more effective it was, and its use as a first-line treatment combined with chemotherapy was superior to its use as a second-line salvage therapy.

\section{The sensitization of first-line immunotherapy IO plus radiotberapy}

In 2019, $7 A M A$ Oncology published two consecutive studies related to the treatment of oligometastatic NSCLC with immunotherapy plus radiotherapy.

(I) A single-arm phase 2 study (25) demonstrated that after the completion of locally ablative therapy (LAT) such as surgery or stereotactic radiotherapy for oligometastatic NSCLC ( $\leq 4$ metastatic sites), the mPFS of patients subsequently treated with pembrolizumab was 19.1 months, significantly 
longer than the historical median of 6.6 months $(\mathrm{P}=0.005)$, with no new safety signals and no reduction in quality of life.

(II) Another important randomized controlled trial, the PembroRT study (26), was designed to evaluate the tolerability and efficacy of SBRT prior to pembrolizumab treatment on a single metastatic site. The ORR of the control arm and the experimental arm was $18 \%(\mathrm{n}=40)$ and $36 \%$ $(\mathrm{n}=36)$, respectively, and the mPFS was 1.9 and 6.6 months, respectively (HR, 0.71; 95\% CI, $0.42-1.18 ; \mathrm{P}=0.19)$. Due to reasons including experimental design, the PembroRT study did not meet its prespecified end point, but it clearly showed the powerful sensitization effect of radiotherapy, which is worthy of further examination.

\section{IO plus chemotherapy}

At present, all of the first-line studies are aiming to compare whether immunotherapy should be added on the basis of chemotherapy. Therefore, it is still controversial whether to add chemotherapy on the basis of immunotherapy for a population which is suitable for immunotherapy, especially for those with PD-L1 expression $>50 \%$. Through an indirect comparison of 11 first-line RCTs, Jianxing He and Wenhua Liang's research group from Guangzhou Institute of Respiratory Health found that adding chemotherapy on the basis of immunotherapy could further significantly improve PFS/OS in the overall population and in the high PD-L1 expression population (27). This provides evidence for treatment selection, whereas the optimal sensitization model still needs to be optimized and confirmed.

PD-1 monoclonal antibody plus CTLA-4 monoclonal antibody In September 2019, NEJM once again published the results from the CheckMate 227 study (28), confirming that for patients with NSCLC, nivolumab plus ipilimumab resulted in a longer OS duration than platinum-containing chemotherapy, providing a "chemo-free" first-line treatment regimen for these patients. The researchers enrolled more than 1,700 untreated advanced NSCLC patients who were EGFR and ALK mutation negative.

First, the patients were stratified according to their expression of PD-L1, and divided into the PD-L1 positive group (PD-L1 expression $\geq 1 \%$ ) and the PD-L1 negative group (PD-L1 expression $<1 \%$ ). Each group was further divided into three subgroups: the PD-L1 positive group was divided into the dual immunotherapy (nivolumab plus ipilimumab), single immunotherapy (nivolumab alone group), and chemotherapy groups (standard chemotherapy regimen). The grouping of the $\mathrm{PD}-\mathrm{L} 1$ negative group was similar to that of the PD-L1 positive group, except the single immunotherapy group was combined with chemotherapy in PD-L1 negative group, while the other two subgroups were the same as the in the PD-L1 positive group.

There were two primary end points: (I) patients with a high TMB (TMB $\geq 10$ mutations per megabase): PFS with dual immunotherapy versus with chemotherapy; and (II) PD-L1 positive patients (PD-L1 expression level of $1 \%$ or more): OS with dual immunotherapy versus with chemotherapy. NEFM reported the PFS in patients with a high TMB in 2018, with the dual immunotherapy group being obviously superior to the chemotherapy group. A second primary end point was simultaneously reported by ESMO congress and NEFM in 2019, showing that the OS of PD-L1 positive patients was significantly longer with dual immunotherapy (NV plus ipilimumab) than with chemotherapy. Furthermore, regardless of the level of PD-L1 expression and TMB, the mOS of the dual immunotherapy group was longer than that of the chemotherapy group, especially in the PD-L1 negative group (17.2 vs. 12.2 months; HR, 0.62). Additionally, the median DoR (mDoR) was also significantly longer in the dual immunotherapy group than in the chemotherapy group (23.2 vs. 6.2 months).

\section{IO plus antiangiogenic agents}

Following the Impower150 study's report in 2018 of the efficacy of chemotherapy + atezolizumab + bevacizumab, a multicenter, phase Ib study that aimed to assess the preliminary antitumor activity of the inhibitor of vascular endothelial growth factor receptor (VEGFR) ramucirumab plus pembrolizumab in multiple types of cancer (including lung cancer) patients was published in The Lancet Oncology in 2019 (29). Patients with NSCLC attained ORR of 30\% and a disease control rate (DCR) of $86 \%$, which suggests this is a regimen worthy of further exploration.

\section{The first-line treatment for EGFR-mutated advanced NSCLC}

The third-generation tyrosine kinase inbibitor of the EGFR (EGFR-TKI) consolidated its status in first-line treatment

NEFM once again published the FLAURA study, the foremost research into targeted therapy of lung cancer (30). This trial was designed to compare the efficacy of thirdgeneration EGFR-TKI osimertinib as the first-line 
treatment with first-generation EGFR-TKI. The analysis of the FLAURA study, which was reported for the first time in 2017, showed that the PFS of the osimertinib group was significantly longer than that of the control group, and the mPFS was 18.9 and 10.2 months (HR, 0.46; $\mathrm{P}<0.001$ ), respectively.

As announced in NEFM and at the ESMO congress 2019 , the mOS of the osimertinib group and the comparator group was 38.6 and 31.8 months, respectively (HR, 0.8; $\mathrm{P}=0.046$ ), achieving a reduction of $20 \%$ in disease mortality risk in the osimertinib group (HR, 0.80; 95\% CI, 0.64-1.00; $\mathrm{P}=0.046)$. The 12-, 24-, and 36-month OS rates in the osimertinib group were all better than those in the first-generation EGFR-TKI group, and osimertinib also extended the time to first and second subsequent therapy. An OS benefit with osimertinib was observed across most of the predefined subgroups, apart from the Asian subgroup (HR, 1.00; 95\% CI, 0.75-1.32).

In addition, Fournal of Clinical Oncology published the results of the BLOOM study, which investigated osimertinib treatment in patients with EGFR-mutated NSCLC and leptomeningeal metastases (LMs) (31). The LM ORR and DoR were 62\% (95\% CI, 45-78\%) and 15.2 months (95\% CI, 7.5-17.5 months), respectively. The FLAURA study established the treatment mode of putting third-generation TKI as a priority option in firstline treatment, but its results in Asian populations remain controversial.

\section{First-generation EGFR-TKI plus chemotherapy}

(I) A phase 3 randomized controlled trial NEJ005 was released at the 2019 ASCO annual meeting, with the purpose of comparing gefitinib plus chemotherapy with gefitinib alone as the firstline treatment (32). In this trial, estimated mPFS was significantly longer with gefitinib plus chemotherapy than gefitinib alone (16 vs. 8 months; HR, 0.5; 95\% CI, 0.39-0.65; $\mathrm{P}<0.001)$. Moreover, patients treated with gefitinib plus chemotherapy had longer mOS than those treated with gefitinib alone (NR vs. 18 months; HR, 0.45; $95 \%$ CI, 0.31-0.66; $\mathrm{P}<0.001)$. In terms of adverse events, the incidence of adverse events $\geq$ grade 3 was $51 \%$ for the gefitinib plus chemotherapy arm and $25 \%$ in the gefitinib group, with significant difference between the arms $(\mathrm{P}<0.001)$. The NEJ009 study presented its results at the ASCO annual meeting 2018, and these two studies were published successively in the Fournal of Clinical Oncology.

(II) At the WCLC conference 2019, Professor Baohui Han and his team from Shanghai Chest Hospital affiliated to Shanghai Jiao Tong University verbally reported the efficacy and safety of firstgeneration EGFR-TKI gefitinib combined with chemotherapy as the first-line treatment for advanced EGFR-mutated NSCLC (33). Updated data of the clinical trial showed that the mOS in the combinational group was obviously extended to 37.9 months (95\% CI:17.3-58.6). Multiple studies have confirmed that first-generation EGFR-TKI combined with chemotherapy shows significant survival benefit. However, the comparison of firstgeneration EGFR-TKI plus chemotherapy with third-generation EGFR-TKI, and the mechanism of first-line treatment resistance are yet to be fully explored.

\section{First-generation EGFR-TKI plus antiangiogenic agents}

(I) The ARTEMIS study is a Chinese multicenter, randomized, controlled phase 3 clinical trial aiming to investigate the efficacy and safety of erlotinib with or without bevacizumab as the first-line treatment for EGFR-mutated NSCLC patients. The study was verbally presented by Professor Qing Zhou from Guangdong Provincial People's Hospital at ESMO congress 2019 (34). The mPFS evaluated by the Independent Review Committee (IRC) was 18 months in the combinational group and 11.3 months in the erlotinib group (HR, 0.55; $\mathrm{P}<0.001$ ), and PFS benefited significantly in the combinational group. In addition, the analysis of drug resistance showed a similar proportion of T790M mutation between these two groups $(\mathrm{P}=0.732)$.

(II) The RELAY study was a worldwide, randomized, double-blind, and placebo-controlled study which evaluated ramucirumab plus erlotinib versus placebo plus erlotinib as first-line treatment for advanced NSCLC patients (35). There was a significantly statistical and clinical improvement of PFS in the treatment group compared to the placebo group (mPFS, 19.4 vs. 12.4 months; HR, $0.59 ; 95 \%$ CI, 0.46-0.79; $\mathrm{P}<0.0001)$, and the frequencies of EGFR-T790M mutation were similar between the groups. The related results were published in the The Lancet Oncology. 
The combination of first-generation EGFR-TKI with antiangiogenic agents can delay the occurrence of drug resistance, but the long-term survival benefit has not yet been confirmed, and, at present, there is not enough evidence which demonstrates that this treatment model can change the biological activity of tumor.

\section{Comprehensive comparison of first-line treatment regimens}

Regarding the numerous options currently available for first-line treatment for EGFR mutations, Jianxing He and Wenhua Liang's research group from Guangzhou Medical University conducted a network meta-analysis involving a total of 4,628 patients from 18 RCTs (36). This metaanalysis found that gefitinib plus chemotherapy and thirdgeneration EGFR-TKI osimertinib ranked highest and equal in efficacy among all regimens. Furthermore, the meta-analysis indicated that these two regimens were the optimal therapy for the two major mutation types of EGFR L858R and exon 19 deletion, respectively.

This study was the first study related to the treatment of lung cancer to be published in the BMF in China, providing evidence for the optimization and individualized options of first-line regimens for patients with EGFR mutations.

\section{Third-generation EGFR-TKI showed efficacy on}

\section{uncommon mutations}

A multicenter, open-label, single-arm, phase II trial published in the Fournal of Clinical Oncology demonstrated that patients with NSCLC harboring EGFR mutations (other than the exon 19 deletion, L858R and T790M mutations, and exon 20 insertion) achieved an ORR of $50 \%$ (95\% CI, 33-67\%, 18/36) after receiving osimertinib treatment (37). The mPFS was 8.2 months (95\% CI, 5.9-10.5 months) and the mDoR was 11.2 months (95\% CI, 7.7-14.7 months), with manageable toxicity.

\section{Anaplastic lymphoma kinase-positive (ALK-positive) advanced NSCLC \\ ALESIA}

The ALESIA study, led by Professor Caicun Zhou from Shanghai Pulmonary Hospital Affiliated to Tongji University School of Medicine, was published in The Lancet Respiratory Medicine (38). This trial aimed to compare the first-line treatment of alectinib and crizotinib head-to-head in Asian patients with advanced ALK-positive NSCLC. The mPFS was NR in the alectinib group compared with 11.1 months in the crizotinib group. (HR, 0.22; 95\% CI, $0.13-0.38 ; \mathrm{P}<0.0001)$. OS data are currently immature for both groups (HR, 0.28; 95\% CI, 0.12-0.68; $\mathrm{P}=0.0027$ ), but the results showed that the prognosis of both groups of patients with or without central nervous system (CNS) metastatic lesions was superior in the alectinib group, and alectinib can significantly delay the progression of CNS (HR, 0.14; 95\% CI, 0.06-0.30). Additionally, the incidence of grade 3-5 adverse events in the alectinib group was lower than that in the crizotinib group.

\section{Ensartinib}

A phase 2 clinical trial led by Professor Li Zhang/Yunpeng Yang from Sun Yat-sen University Cancer Center which assessed the efficacy and safety of ensartinib in ALKpositive NSCLC patients who underwent crizotinib treatment failure was published in The Lancet Respiratory Medicine (39). A total of 156 patients, $62 \%$ of whom had $\mathrm{BMs}$, were included in the final analysis. Data showed that $52 \%$ of patients attained a response after treatment, the DCR reached $93 \%$, and the mPFS was 9.6 months. Among the patients with BMs, the intracranial ORR was $70 \%$, and the DCR of intracranial lesions reached $98 \%$. Moreover, ensartinib had significant efficacy with varieties of ALKresistant mutations, including those of other secondgeneration ALK inhibitors.

\section{ROS1 fusion-positive advanced NSCLC Entrectinib}

An integrated analysis of three phase 1-2 clinical trials published in The Lancet Oncology showed that entrectinib was effective and well tolerated for advanced ROS1-fusion positive NSCLC (40). The outcomes from trials ALKA372-001, STARTRK-1, and STARTRK-2 indicated that the ORR of 53 ROS1-fusion positive NSCLC patients treated with entrectinib was $77 \%$, the mPFS was 19 months, and the mDoR was 24.6 months.

\section{Lorlatinib}

The Lancet Oncology published the results of a phase 1-2 clinical trial of lorlatinib in advanced ROS1-positive NSCLC which included a total of 69 ROS1-positive NSCLC patients (41). For 21 TKI-naive patients who had not received ROS1 inhibitors treatment before, the ORR and DCR were $62 \%$ and $90 \%$, respectively, and the $\mathrm{mPFS}$ and $\mathrm{mDoR}$ were 21 and 25.3 months, respectively. Among the 11 patients with CNS metastases, the overall ORR was $45 \%$, the intracranial response rate was $64 \%$, and the median intracranial DoR was not reached. For the 40 patients previously treated with crizotinib as their only TKI, the ORR and DCR were $35 \%$, and $75 \%$, respectively, and the mPFS and mDoR were 8.5 and 13.8 months, respectively. Among the 24 patients with CNS metastases, the ORR rate was $25 \%$, the intracranial response rate was 
$50 \%$, and the median intracranial DoR was not reached.

\section{Advanced NSCLC with KRAS mutation AMG510}

A study evaluating the efficacy of AMG 510 in patients with advanced KRAS G12C mutant solid tumors was reported at ASCO annual meeting 2019 (42). Tumor response was assessable in 29 patients, including 10 NSCLC and 19 colorectal cancer (CRC) patients. All of these patients had previously received $\geq 2$-line treatment regimens. The results indicated that the ORR and DCR of the patients overall were $17.24 \%$ and $79.31 \%$, respectively, and the response lasted for a long duration. In particular, in the ten NSCLC patients, the ORR was $50 \%$ and the DCR reached $100 \%$.

\section{Advanced NSCLC with other mutations Tepotinib}

A study evaluating the efficacy and safety of a novel MET (MET exon 14 skipping mutations) inhibitor tepotinib in advanced NSCLC was presented at the 2019 ASCO annual meeting (43). This single-arm, phase 2 clinical trial was planned to enroll more than 120 patients, who would receive tepotinib $500 \mathrm{mg}$ once a day. At the time the data was released, 85 patients were enrolled, including 55 identified by LBx and 52 by tumor biopsy (TBx). A total of 35 and 41 patients in these two groups were evaluable for efficacy ( $\geq 2$ post-baseline assessments or discontinuation for any reason), respectively. The ORR of the two groups assessed by IRC was $51.4 \%$ and $41.5 \%$, respectively, and the $\mathrm{mDoR}$ was 9.8 and 12.4 months, respectively.

\section{U3-1402}

A phase 1 clinical trial was reported at the 2019 ASCO annual meeting (44). It included patients with advanced EGFR-mutated NSCLC who had previously experienced disease progression after receiving the treatment of EGFR-TKI (including first-/second-generation EGFRTKI and osimertinib) and were subsequently treated with U3-1402. The outcome showed that out of 13 evaluable patients, all but 1 had a decrease in sum of longest diameter of lesions (median best change $-29 \%$, range, $+10 \%$ to $-67 \%$ ), and the DCR reached $100 \%$. In addition, U3-1402 was also effective on different pathways which were activated secondary to EGFR-TKI resistance, including EGFR C797S, HER2, and CDK4.

\section{Entrectinib}

An integrated analysis of phase 1-2 clinical trials was published in The Lancet Oncology and showed that entrectinib was effective and well tolerated in advanced NTRK fusion- positive NSCLC patients (45). Among 54 NTRK fusionpositive NSCLC patients, 31 (57\%; 95\% CI, 43.2-70.8\%) patients had an objective response, with an mPFS of 11.2 months and mDoR of 10.4 months.

\section{TAK-788}

The 2019 ASCO annual meeting reported the efficacy and safety of TAK-788 (an inhibitor of EGFR/HER2) in advanced NSCLC patients with EGFR exon 20 insertions (46). In a phase $1 / 2$ multicenter clinical study, patients who met the inclusion criteria were first subjected to dose escalation and then an expansion cohort. A total of 101 patients were enrolled in the study, $76 \%$ of whom had previously received at least two therapies, and $53 \%$ of whom had BMs. The treatment dose was $5-180 \mathrm{mg}$ once a day, and the recommended phase 2 dose (RP2D) was $160 \mathrm{mg}$ once a day. Twenty-eight patients received this dose (during dose escalation or in the expansion cohort). The ORR and DCR of 26 patients in the extended cohort were 54\% (95\% CI, $33-73 \%$ ) and 89\% (95\% CI, 70-98\%), respectively.

\section{BLU-667}

BLU-667 is a highly potent and selective RET inhibitor, and the data of its efficacy and safety was released at the 2019 ASCO annual meeting (47). A total of 79 patients (21 of dose escalation and 58 of dose expansion) were enrolled and treated with BLU-667, and 39\% of these patients had baseline BMs. Among the 57 patients who received at least one follow-up disease assessment, the ORR was $56 \%$ (95\% CI, 42-69\%), the DCR was $91 \%$, and 6 patients had a DoR of more than 6 months. Among 30 patients treated at the RP2D, the ORR was $60 \%$, and responses occurred regardless of prior treatment or RET fusion genotypes. Only $3 \%$ of patients discontinued because of the adverse event of drug.

\section{Small-cell lung cancer (SCLC)}

The CASPIAN study was a randomized, open-label, worldwide, multicenter phase 3 clinical trial that aimed to explore the efficacy of anti-PD-L1 antibody durvalumab, with or without anti-CTLA-4 antibody tremelimumab, in combination with etoposide plus either cisplatin or carboplatin (platinum-etoposide) in treatment-naive patients with extensive-stage SCLC (ES-SCLC). The results, which were published in The Lancet (48), demonstrated that the mOS was significantly longer in the durvalumab plus platinum-etoposide group compared to the platinumetoposide alone group (13.0 vs. 10.3 months; HR, 0.73; 95\% CI, 0.59-0.91; $\mathrm{P}=0.0047$ ).

To date, there are two PD-L1 monoclonal antibodies 
(atezolizumab and durvalumab) which have obtained positive results in first-line combination treatment of SCLC, establishing the special status of PD-L1 monoclonal antibodies in the treatment of SCLC.

\section{Related research on PD-1 antibodies independently developed in China for lung cancer}

The Chinese-developed PD-1 antibody and related research have entered onto the international stage Camrelizumab

(I) SHR-1210-303 was an open, randomized, multicenter phase 3 clinical trial presented at the WCLC conference 2019 (49), which included patients with advanced non-squamous NSCLC with negative EGFR or ALK and was designed to explore the efficacy of adding camrelizumab (200 mg) on the basis of chemotherapy. The mPFS was 11.3 months (95\% CI, 9.5-NR) in the camrelizumab plus chemotherapy arm and 8.3 months (95\% CI, 6.0-9.7) in the chemotherapy arm (HR, 0.61; 95\% CI, 0.46-0.80; $\mathrm{P}=0.0002)$. For other secondary endpoints including ORR, DCR, DoR, and OS, related data of the camrelizumab plus chemotherapy arm were also superior to the chemotherapy arm.

(II) Another study reported at the 2019 ASCO annual meeting showed that for nonsquamous NSCLC patients treated with camrelizumab plus apatinib as second-line and above treatment, the ORR and DCR in 91 evaluable patients were $29.7 \%$ and $81.3 \%$, respectively, providing a "chemofree" option for patients who received second-line treatment (50).

\section{Sintilimab}

(I) At the WCLC conference 2019, Professor Baohui Han from Shanghai Chest Hospital affiliated to Shanghai Jiao Tong University reported the relevant data of PD-1 monoclonal antibody sintilimab combined with anlotinib in the first-line treatment of advanced NSCLC (51). Treatmentnaive, stage IIIB/IV NSCLC patients were eligible, and patients with EGFR, ALK or ROS1 mutations were excluded. A total of 22 patients were enrolled in this study, and the results showed that the ORR was $68.2 \%$, the DCR was $100 \%$. At the data cutoff, $81.8 \%$ of the patients were still receiving treatment; the proportion of patients with PFS of at least 6 months was $93.8 \%$.

(II) At the WCLC conference 2019, Professors Jie He and Shugeng Gao from Cancer Hospital, Chinese Academy of Medical Sciences released the research results of sintilimab in resectable NSCLC as a neoadjuvant therapy (52). The MPR reached $40.5 \%$ and the pCR reached $16.2 \%$, with manageable safety. This study also designed three postoperative adjuvant treatment cohorts (chemotherapy, PD-1 antibody, and chemotherapy plus PD-1 antibody) synchronously, which are expected to answer the question of postoperative treatment options.

(III) Jianxing He and Wenhua Liang's research group from Guangzhou Institute of Respiratory Health launched a clinical study (CCTC-1901) in relation to sintilimab treatment in early-stage multiple primary lung cancer (MPLC) with ground-glass density (53). This is a single-center, single-arm, prospective Simon's two-stage design phase II clinical trial, aimed at assessing the efficacy of sintilimab in very early-stage ("stage 0") lung cancer.

\section{Toripalimab}

At the WCLC 2019 conference, Professor Caicun Zhou from Shanghai Pulmonary Hospital presented the latest results of a study on toripalimab plus chemotherapy in advanced NSCLC patients with EGFR activating mutations for whom prior EGFR-TKI therapies have failed (54). A total of 40 patients are included in this study and the ORR reached $32.5 \%$ at the 12 th week. As of July 25, 2019, the ORR was $50.0 \%$, the DCR was $87.5 \%$, PR had occurred in 20 patients, 15 patients (including 1 unconfirmed PR) performed SD, the mDoR was 7.0 months, and the mPFS was 7.0 months.

\section{Biomarkers for the drug efficacy}

(I) In a study published in $\mathcal{F} A M A$ (55), Flatiron Health and Foundation Medicine (FMI) confirmed the associations between clinical and genomic characteristics of NSCLC patients through the data of real-world study and demonstrated that targeted therapy could prolong the OS of patients with driver mutations compared to without targeted therapy (18.6 vs. 11.4 months; $\mathrm{P}<0.001$ ), and that patients with TMB-high (TMB-H, $\geq 20$ mutations/ $\mathrm{Mb}$ ) attained longer OS after receiving IO therapy compared to the patients with TMBlow/intermediate (TMB-L/I, <20 mutations/Mb) (16.8 vs. 8.5 months). 
(II) An analysis of the FDA database was published in $7 A M A$ Oncology (56), indicating that a lung immune prognostic index (LIPI) derived from neutrophils/ (leukocytes minus neutrophils) ratio (dNLR) and lactate dehydrogenase (LDH) was significantly correlated with the PFS and OS of immune checkpoint inhibitor (ICI) therapy, targeted therapy, and chemotherapy in advanced NSCLC patients.

(III) Professor Jie Wang and Zhijie Wang from Cancer Hospital, Chinese Academy of Medical Sciences evaluated the feasibility of blood-based TMB (bTMB) as a predictor of efficacy in advanced NSCLC treated with immunotherapy in the Chinese population (57). The results showed that the response rate and response duration of advanced NSCLC patients treated with anti-PD-1/ PD-L1 monoclonal antibody could be predicted by adopting a NCC-GP150 panel based on 150 genes to estimate bTMB based on ctDNA.

\section{Conclusions}

With the incidence of lung cancer increasing, various centers worldwide have gradually started to value early screening and diagnosis of lung cancer. The survival of lung cancer patients has significantly improved compared with historical controls, turning lung cancer from an incurable disease into a chronic disease and suggesting that lung cancer prevention and control need to be carried out with reference to the management mode of common diseases, including popular science and screening activities. Systemic therapy, especially immunotherapy in stage I-III lung cancer, reached a new milestone. Treatment of lung cancer tended to be comprehensive, diversified, and individualized. It is of great importance to explore a biomarker that can accurately predict the efficacy of immunotherapy in the treatment of lung cancer patients. In addition, the mechanism of drug resistance in targeted therapy and how to overcome it is deserving of further investigation in the future.

\section{Acknowledgments}

Funding: None.

\section{Footnote}

Conflicts of Interest: $\mathrm{JH}$ serves as the unpaid Executive Editor-in-Chief and WL serves as the unpaid editorial board member of Fournal of Thoracic Disease. The other authors have no conflicts of interest to declare.

Ethical Statement: The authors are accountable for all aspects of the work in ensuring that questions related to the accuracy or integrity of any part of the work are appropriately investigated and resolved.

Open Access Statement: This is an Open Access article distributed in accordance with the Creative Commons Attribution-NonCommercial-NoDerivs 4.0 International License (CC BY-NC-ND 4.0), which permits the noncommercial replication and distribution of the article with the strict proviso that no changes or edits are made and the original work is properly cited (including links to both the formal publication through the relevant DOI and the license). See: https://creativecommons.org/licenses/by-nc-nd/4.0/.

\section{References}

1. Zeng H, Chen W, Zheng R, et al. Changing cancer survival in China during 2003-15: a pooled analysis of 17 population-based cancer registries. Lancet Glob Health 2018;6:e555-67.

2. Zhou M, Wang H, Zeng X, et al. Mortality, morbidity, and risk factors in China and its provinces, 1990-2017: a systematic analysis for the Global Burden of Disease Study 2017. Lancet 2019;394:1145-58.

3. Yang JJ, Yu D, Xiang YB, et al. Association of Dietary Fiber and Yogurt Consumption With Lung Cancer Risk: A Pooled Analysis. JAMA Oncol 2019. [Epub ahead of print].

4. Dai J, Lv J, Zhu M, et al. Identification of risk loci and a polygenic risk score for lung cancer: a large-scale prospective cohort study in Chinese populations. Lancet Respir Med 2019;7:881-91.

5. Cohn AL, Seiden M, Kurtzman KN, et al. The Circulating Cell-free Genome Atlas (CCGA) Study: Follow-up (F/U) on non-cancer participants with cancer-like cell-free DNA signals. J Clin Oncol 2019;37:abstr 5574.

6. Oxnard GR, Klein EA, Seiden MV, et al. Simultaneous multi-cancer detection and tissue of origin (TOO) localization using targeted bisulfite sequencing of plasma cell-free DNA (cfDNA). Ann Oncol 2019;30:912.

7. Liang $\mathrm{W}$, Zhao $\mathrm{Y}$, Huang $\mathrm{W}$, et al. Non-invasive diagnosis of early-stage lung cancer using high-throughput targeted DNA methylation sequencing of circulating tumor DNA (ctDNA). Theranostics 2019;9:2056-70. 
8. Kwiatkowski DJ, Rusch VW, Chaft JE, et al. Neoadjuvant atezolizumab in resectable non-small cell lung cancer (NSCLC): Interim analysis and biomarker data from a multicenter study (LCMC3). J Clin Oncol 2019;37:abstr 8503 .

9. Cascone T, William WN, Weissferdt A, et al. Neoadjuvant nivolumab $(\mathrm{N})$ or nivolumab plus ipilimumab (NI) for resectable non-small cell lung cancer (NSCLC): Clinical and correlative results from the NEOSTAR study. J Clin Oncol 2019;37:abstr 8504.

10. Provencio M, Nadal E, Insa A, et al. Neoadjuvant chemo-immunotherapy for the treatment of stage IIIA resectable non-small-cell lung cancer (NSCLC): A phase II multicenter exploratory study-Final data of patients who underwent surgical assessment. J Clin Oncol 2019;37:abstr 8509.

11. Ball D, Mai GT, Vinod S, et al. Stereotactic ablative radiotherapy versus standard radiotherapy in stage 1 nonsmall-cell lung cancer (TROG 09.02 CHISEL): a phase 3 , open-label, randomised controlled trial. Lancet Oncol 2019;20:494-503.

12. Zeng Y, Mayne N, Yang CJ, et al. A Nomogram for Predicting Cancer-Specific Survival of TNM 8th Edition Stage I Non-small-cell Lung Cancer. Ann Surg Oncol 2019;26:2053-62.

13. Zhao Y, Wang W, Liang H, et al. The Optimal Treatment for Stage IIIA-N2 Non-Small Cell Lung Cancer: A Network Meta-Analysis. Ann Thorac Surg 2019;107:1866-75.

14. Gray JE, Villegas AE, Daniel DB, et al. Three-year overall survival update from the PACIFIC trial. J Clin Oncol 2019;37:abstr 8526.

15. Hui R, Özgüroğlu M, Villegas A, et al. Patient-reported outcomes with durvalumab after chemoradiotherapy in stage III, unresectable non-small-cell lung cancer (PACIFIC): a randomised, controlled, phase 3 study. Lancet Oncol 2019;20:1670-80.

16. Sun A, Hu C, Wong SJ, et al. Prophylactic Cranial Irradiation vs Observation in Patients With Locally Advanced Non-Small Cell Lung Cancer: A Long-term Update of the NRG Oncology/RTOG 0214 Phase 3 Randomized Clinical Trial. JAMA Oncol 2019;5:847-55.

17. Reck M, Rodriguez-Abreu D, Robinson AG, et al. KEYNOTE-024 3-Year Survival Update: Pembrolizumab vs Platinum-Based Chemotherapy for Advanced Non-Small-Cell Lung Cancer. J Thorac Oncol 2019;14:S243.

18. Mok TSK, Wu YL, Kudaba I, et al. Pembrolizumab versus chemotherapy for previously untreated, PD-L1expressing, locally advanced or metastatic non-small-cell lung cancer (KEYNOTE-042): a randomised, open-label, controlled, phase 3 trial. Lancet 2019;393:1819-30.

19. Spigel D, de Marinis F, Giaccone G, et al. IMpower110: Interim overall survival (OS) analysis of a phase III study of atezolizumab (atezo) vs platinum-based chemotherapy (chemo) as first-line (1L) treatment (tx) in PD-L1-selected NSCLC. Ann Oncol 2019;30:915.

20. Antonia SJ, Borghaei H, Ramalingam SS, et al. Four-year survival with nivolumab in patients with previously treated advanced non-small-cell lung cancer: a pooled analysis. Lancet Oncol 2019;20:1395-408.

21. Garon EB, Hellmann MD, Costa EC, et al. Five-year long-term overall survival for patients with advanced NSCLC treated with pembrolizumab: Results from KEYNOTE-001. J Clin Oncol 2019;37:LBA9015.

22. Gadgeel SM, Garassino MC, Esteban E, et al. KEYNOTE-189: Updated OS and progression after the next line of therapy (PFS2) with pembrolizumab (pembro) plus chemo with pemetrexed and platinum vs placebo plus chemo for metastatic nonsquamous NSCLC. J Clin Oncol 2019;37:abstr 9013.

23. Paz-Ares L, Vicente D, Tafreshi A, et al. LBA82 Pembrolizumab (pembro) + chemotherapy (chemo) in metastatic squamous NSCLC: Final analysis and progression after the next line of therapy (PFS2) in KEYNOTE-407. Ann Oncol 2019;30:v918-9.

24. West H, McCleod M, Hussein M, et al. Atezolizumab in combination with carboplatin plus nab-paclitaxel chemotherapy compared with chemotherapy alone as firstline treatment for metastatic non-squamous non-smallcell lung cancer (IMpower130): a multicentre, randomised, open-label, phase 3 trial. Lancet Oncol 2019;20:924-37.

25. Bauml JM, Mick R, Ciunci C, et al. Pembrolizumab After Completion of Locally Ablative Therapy for Oligometastatic Non-Small Cell Lung Cancer: A Phase 2 Trial. JAMA Oncol 2019. [Epub ahead of print].

26. Theelen WSME, Peulen HMU, Lalezari F, et al. Effect of Pembrolizumab After Stereotactic Body Radiotherapy vs Pembrolizumab Alone on Tumor Response in Patients With Advanced Non-Small Cell Lung Cancer: Results of the PEMBRO-RT Phase 2 Randomized Clinical Trial. JAMA Oncol 2019. [Epub ahead of print].

27. Liang H, Liu Z, Cai X, et al. PD-(L)1 inhibitors vs. chemotherapy vs. their combination in front-line treatment for NSCLC: An indirect comparison. Int J Cancer 2019;145:3011-21. 
28. Hellmann MD, Paz-Ares L, Bernabe Caro R, et al. Nivolumab plus Ipilimumab in Advanced Non-Small-Cell Lung Cancer. N Engl J Med 2019;381:2020-31.

29. Herbst RS, Arkenau HT, Santana-Davila R, et al. Ramucirumab plus pembrolizumab in patients with previously treated advanced non-small-cell lung cancer, gastro-oesophageal cancer, or urothelial carcinomas (JVDF): a multicohort, non-randomised, open-label, phase 1a/b trial. Lancet Oncol 2019;20:1109-23.

30. Ramalingam SS, Vansteenkiste J, Planchard D, et al. Overall Survival with Osimertinib in Untreated, EGFR-Mutated Advanced NSCLC. N Engl J Med 2020;382:41-50.

31. Yang JCH, Kim SW, Kim DW, et al. Osimertinib in Patients With Epidermal Growth Factor Receptor Mutation-Positive Non-Small-Cell Lung Cancer and Leptomeningeal Metastases: The BLOOM Study. J Clin Oncol 2020;38:538-47.

32. Noronha V, Joshi A, Patil VM, et al. Phase III randomized trial comparing gefitinib to gefitinib with pemetrexedcarboplatin chemotherapy in patients with advanced untreated EGFR mutant non-small cell lung cancer (gef vs gef+C). J Clin Oncol 2019;37:abstr 9001.

33. Zhang W, Xu J, Lou Y, et al. Chemotherapy Plus EGFRTKI as First-Line Treatment Provides Better Survival for EGFR Mutation NSCLC Patients: Update Data for NCT02148380. J Thorac Oncol 2019;14:S236.

34. Zhou Q, Wu YL, Cheng Y, et al. 1480 O - CTONG 1509: Phase III study of bevacizumab with or without erlotinib in untreated Chinese patients with advanced EGFR-mutated NSCLC. Ann Oncol 2019;30:v603.

35. Nakagawa K, Garon EB, Seto T, et al. Ramucirumab plus erlotinib in patients with untreated, EGFRmutated, advanced non-small-cell lung cancer (RELAY): a randomised, double-blind, placebo-controlled, phase 3 trial. Lancet Oncol 2019;20:1655-69.

36. Zhao Y, Liu J, Cai X, et al. Efficacy and safety of first line treatments for patients with advanced epidermal growth factor receptor mutated, non-small cell lung cancer: systematic review and network meta-analysis. BMJ 2019;367:15460.

37. Cho JH, Lim SH, An HJ, et al. Osimertinib for Patients With Non-Small-Cell Lung Cancer Harboring Uncommon EGFR Mutations: A Multicenter, OpenLabel, Phase II Trial (KCSG-LU15-09). J Clin Oncol 2020;38:488-95.

38. Zhou C, Kim SW, Reungwetwattana T, et al. Alectinib versus crizotinib in untreated Asian patients with anaplastic lymphoma kinase-positive non-small-cell lung cancer (ALESIA): a randomised phase 3 study. Lancet Respir Med 2019;7:437-46.

39. Yang Y, Zhou J, Zhou J, et al. Efficacy, safety, and biomarker analysis of ensartinib in crizotinib-resistant, ALK-positive non-small-cell lung cancer: a multicentre, phase 2 trial. Lancet Respir Med 2020;8:45-53.

40. Drilon A, Siena S, Dziadziuszko R, et al. Entrectinib in ROS1 fusion-positive non-small-cell lung cancer: integrated analysis of three phase 1-2 trials. Lancet Oncol 2020;21:261-70.

41. Shaw AT, Solomon BJ, Chiari R, et al. Lorlatinib in advanced ROS1-positive non-small-cell lung cancer: a multicentre, open-label, single-arm, phase 1-2 trial. Lancet Oncol 2019;20:1691-701.

42. Fakih M, O'Neil B, Price TJ, et al. Phase 1 study evaluating the safety, tolerability, pharmacokinetics (PK), and efficacy of AMG 510, a novel small molecule KRASG12C inhibitor, in advanced solid tumors. J Clin Oncol 2019;37:abstr 3003.

43. Paik PK, Veillon R, Cortot AB, et al. Phase II study of tepotinib in NSCLC patients with METex14 mutations. J Clin Oncol 2019;37:abstr 9005.

44. Janne PA, Yu HA, Johnson ML, et al. Safety and preliminary antitumor activity of U3-1402: A HER3targeted antibody drug conjugate in EGFR TKI-resistant, EGFRm NSCLC. J Clin Oncol 2019;37:abstr 9010.

45. Doebele RC, Drilon A, Paz-Ares L, et al. Entrectinib in patients with advanced or metastatic NTRK fusionpositive solid tumours: integrated analysis of three phase 1-2 trials. Lancet Oncol 2020;21:271-82.

46. Janne PA, Neal JW, Camidge DR, et al. Antitumor activity of TAK-788 in NSCLC with EGFR exon 20 insertions. J Clin Oncol 2019;37:abstr 9007.

47. Gainor JF, Lee DH, Curigliano G, et al. Clinical activity and tolerability of BLU-667, a highly potent and selective RET inhibitor, in patients (pts) with advanced RETfusion+ non-small cell lung cancer (NSCLC). J Clin Oncol 2019;37:abstr 9008.

48. Paz-Ares L, Dvorkin M, Chen Y, et al. Durvalumab plus platinum-etoposide versus platinum-etoposide in firstline treatment of extensive-stage small-cell lung cancer (CASPIAN): a randomised, controlled, open-label, phase 3 trial. Lancet 2019;394:1929-39.

49. Zhou C, Chen G, Huang Y, et al. OA04.03 A Randomized Phase 3 Study of Camrelizumab plus Chemotherapy as 1st Line Therapy for Advanced/Metastatic NonSquamous Non-Small Cell Lung Cancer. J Thorac Oncol 
2019;14:S215-6.

50. Zhou C, Gao G, Wang YN, et al. Efficacy of PD-1 monoclonal antibody SHR-1210 plus apatinib in patients with advanced nonsquamous NSCLC with wild-type EGFR and ALK. J Clin Oncol 2019;37:abstr 9112.

51. Han B, Chu T, Zhong R, et al. JCSE01.11 Efficacy and Safety of Sintilimab with Anlotinib as First-Line Therapy for Advanced Non-Small Cell Lung Cancer (NSCLC). J Thorac Oncol 2019;14:S129.

52. Li N, Ying J, Tao X, et al. Efficacy and Safety of Neoadjuvant PD-1 Blockade with Sintilimab in Resectable Non-Small Cell Lung Cancer. J Thorac Oncol 2019;14:S627-8.

53. Editorial Office. The first attempt worldwide to test PD-1 antibody on multiple "Stage 0" lung cancer. Transl Lung Cancer Res 2019;8:556-7.

54. Zhang J, Zhou C, Zhao Y, et al. MA11.06 A PII Study of Toripalimab, a PD-1 mAb, in Combination with

Cite this article as: Cheng B, Xiong S, Li C, Liang H, Zhao Y, Li J, Shi J, Ou L, Chen Z, Liang P, Liang W, He J. An annual review of the remarkable advances in lung cancer clinical research in 2019. J Thorac Dis 2020;12(3):1056-1069. doi: $10.21037 /$ jtd. 2020.03 .11
Chemotherapy in EGFR+ Advanced NSCLC Patients Failed to Prior EGFR TKI Therapies. J Thorac Oncol 2019; 14:S292.

55. Singal G, Miller PG, Agarwala V, et al. Association of Patient Characteristics and Tumor Genomics With Clinical Outcomes Among Patients With Non-Small Cell Lung Cancer Using a Clinicogenomic Database. JAMA 2019;321:1391-9.

56. Mezquita L, Auclin E, Ferrara R, et al. Association of the Lung Immune Prognostic Index With Immune Checkpoint Inhibitor Outcomes in Patients With Advanced Non-Small Cell Lung Cancer. JAMA Oncol 2018;4:351-7.

57. Wang Z, Duan J, Wang G, et al. Allele FrequencyAdjusted Blood-Based Tumor Mutational Burden as a Predictor of Overall Survival for Patients With NSCLC Treated With PD-(L)1 Inhibitors. J Thorac Oncol 2019. [Epub ahead of print]. 